\title{
A tangential differential operator applied to stress and traction boundary integral equations for plate bending including the shear deformation effect
}

\author{
L. Palermo Jr. \\ Faculty of Civil Engineering, Architecture and Urban Design, \\ State University of Campinas, Brazil
}

\begin{abstract}
Stress boundary integral equations (BIEs) are required in elastic or inelastic analyses of plate bending problems to obtain distributed shear, bending and twisting moments. Traction BIE, which is important to perform fracture analyses, is directly related to stress BIE. The collocation point position and the strategy to treat improper integrals are essential features studied in BIE for tractions or stresses at boundary points. The tangential differential operator (TDO) is used in stress and traction BIEs to reduce the strong singularities in the fundamental solution kernels and remaining singularities can be treated with the Cauchy principal value sense or the first order regularization. This study presents the application of the TDO for stress and traction BIEs used in plate bending models considering the shear deformation effect. The results in bending problems are obtained with traction BIE using TDO, instead of displacement $\mathrm{BIE}$, and are compared to those in the literature where the problem was solved with traction BIE containing the strong singularity or with displacement BIE.
\end{abstract}

\section{Introduction}

Distributed shear, bending and twisting moments required in plate bending analyses are computed with stress BIEs. The differentiation in the fundamental solution kernels of displacement BIE, to obtain BIEs for stresses, increases the order of singularities. Strong singularities appear in fundamental solution kernels of stress BIE when values at boundary points are required as well as in those of 
traction BIE because these equations are related according to the Cauchy formula. The collocation point position and the strategy to treat improper integrals are the essential features studied in numerical implementations for traction or stress BIE in plate bending or in elasticity $[1,2]$. The use of the tangential differential operator (TDO) in conjunction with the integration by parts is a way to reduce the order of singularities in stress or traction BIE when Kelvin type fundamental solutions are used. Kupradze [3] first presented an application using the tangential differential operator (TDO) and Sladek and Sladek [4] employed the TDO in a curved crack solution. Regularized boundary element formulations employing TDO for potential and elasticity problems, including fracture mechanics formulations, were presented by Bonnet in [5]. The dual boundary element formulation for two dimensional problems of linear fracture mechanics using TDO in traction BIE was studied in [6]. The strategy presented in [6], which allowed the application of TDO in problems using non-conformal interpolations, was extended in [7] for the traction BIE in three dimensional elasticity.

The purpose of this study is introducing the TDO in stress and traction BIEs for plate bending models including the shear deformation effect. Kelvin type fundamental solution is the main requirement to apply the TDO in conjunction with the integration by parts to reduce singularities in BIE. The motivation to apply the TDO in plate bending models lies on developed studies for [6]. The efficiency of stress BIE for two dimensional elasticity problems was improved when the strong singularity was reduced [8] and the main result was the application for fracture problems in [6]. It is necessary to note the boundary element meshes and positions for collocation points used in [6] were first tested for traction BIEs containing strong singularities in [9] and the results were not changed with reference to those available in the literature. The application of the TDO for plate bending models is similar but not equal to the algebraic manipulation presented in [6] or [7]. The plate bending model represents the equilibrium of out of plane loads by in-plane stresses non-uniformly distributed on the thickness [10].

The boundary element formulation for plates using the TDO in BIE for stresses is extended to traction BIE. TDO was applied in the fundamental solution kernel with the strong singularity whereas other kernels remain unchanged. The results in bending problems are obtained with the traction BIE using TDO instead of displacement BIE and they are compared to those in the literature where the problem was solved with traction BIE containing the strong singularity or with displacement BIE. Fracture problems using the dual boundary element method (DBEM) were not considered because results in DBEM can be changed, too, according to the collocation point position of the displacement BIE, as shown in [11]. Thus, fracture problems will be analyzed in other study.

\section{Application of the tangential differential operator}

The equilibrium equations for an infinitesimal plate element under a transverse distributed loading $\mathrm{q}\left(\mathrm{x}_{\mathrm{i}}\right)$ are next written with Latin indices considering values $\{1,2$ and 3$\}$ and Greek indices considering values $\{1,2\}$ : 


$$
\begin{gathered}
\mathrm{M}_{\alpha \beta, \beta}-\mathrm{Q}_{\alpha}=0 \\
\mathrm{Q}_{\alpha, \alpha}+\mathrm{q}=0
\end{gathered}
$$

The plate has uniform thickness $\mathrm{h}$ and the constitutive relations are next written using a unified notation for the Reissner [12] and the Mindlin [13] model:

$$
\begin{gathered}
\mathrm{M}_{\alpha \beta}=\mathrm{D} \frac{1-v}{2}\left(\psi_{\alpha, \beta}+\psi_{\beta, \alpha}+\frac{2 v}{1-v} \psi_{\gamma, \gamma} \delta_{\alpha \beta}\right)+\delta_{\alpha \beta} \mathrm{qRE} \\
\mathrm{Q}_{\alpha}=\mathrm{D} \frac{1-v}{2} \lambda^{2}\left(\psi_{\alpha}+\mathrm{w}_{, \alpha}\right)
\end{gathered}
$$

with

$$
\begin{aligned}
\lambda^{2} & =12 \frac{\kappa^{2}}{h^{2}} \\
\mathrm{RE} & =\frac{v}{\lambda^{2}(1-v)}
\end{aligned}
$$

$\mathrm{D}$ is the flexural rigidity, $v$ is the Poisson ratio, $\mathrm{w}$ is the deflection, $\psi_{\alpha}$ is the plate rotation in the direction $\alpha, \delta_{\alpha \beta}$ is the Kronecker delta. The product qRE in equation (3) corresponds to the linearly weighted average effect of the normal stress component in the thickness direction and should be is considered in the Reissner model but not in the Mindlin model, when it should be considered null in equation (3). Shear parameter $\kappa^{2}$ is equal to $5 / 6$ and $\pi^{2} / 12$ for the Reissner and the Mindlin model, respectively.

A unified displacement BIE for the Reissner and the Mindlin model can be written in terms of generalized displacements and tractions presented in [14]:

$$
\mathrm{C}_{\mathrm{ij}} \mathrm{u}_{\mathrm{j}}+\oint_{\Gamma}\left(\mathrm{T}_{\mathrm{j}}^{\mathrm{i}} \mathrm{u}_{\mathrm{j}}-\mathrm{U}_{\mathrm{j}}^{\mathrm{i}} \mathrm{t}_{\mathrm{j}}\right) \mathrm{d} \Gamma=\iint_{\Omega} \mathrm{q}\left(\mathrm{U}_{3}^{\mathrm{i}}-\mathrm{U}_{\alpha, \alpha}^{\mathrm{i}} \mathrm{RE}\right) \mathrm{d} \Omega
$$

where $\mathrm{u}_{\alpha}$ is $\psi_{\alpha}, \mathrm{u}_{3}$ is $\mathrm{w}, \mathrm{t}_{\alpha}$ is the product $\mathrm{M}_{\alpha \beta} \cdot \mathrm{n}_{\beta}, \mathrm{t}_{3}$ is the product $\mathrm{Q}_{\alpha} \cdot \mathrm{n}_{\alpha} \cdot \mathrm{U}_{\mathrm{j}}^{\mathrm{i}}$ represents the rotation $(j=1,2)$ or the deflection $(j=3)$ due to a unit couple $(i=1,2)$ or a unit point force $(\mathrm{i}=3) . \mathrm{C}_{\mathrm{ij}}$ is an element of the matrix $\mathrm{C}$ related to the collocation point position that makes a diagonal matrix with elements equal to 1 for internal collocation points or equal to 0.5 for collocation points on a smooth boundary. The integrand of the domain integral in equation (7) contains the RE factor, which should be cut off for analyses using the Mindlin model.

The application of the TDO will be next presented using Mindlin's model to simplify the presentation because the difference with reference to Reissner's model in the boundary element method corresponds to the introduction of RE parameter which is in an additional domain integral, as shown in equation (7) for the displacement BIE. The BIE for the deflection gradient at an internal point can be written using the differentiation in terms of field variables:

$$
\begin{aligned}
\mathrm{u}_{3, \gamma}= & \oint_{\Gamma}\left(\frac{\partial}{\partial \mathrm{x}_{\gamma}}\left(\mathrm{T}_{\beta}^{3}\right) \mathrm{u}_{\beta}+\frac{\partial}{\partial \mathrm{x}_{\gamma}}\left(\mathrm{T}_{3}^{3}\right) \mathrm{u}_{3}-\frac{\partial}{\partial \mathrm{x}_{\gamma}}\left(\mathrm{U}_{\beta}^{3}\right) \mathrm{t}_{\beta}-\frac{\partial}{\partial \mathrm{x}_{\gamma}}\left(\mathrm{U}_{3}^{3}\right) \mathrm{t}_{3}\right) \mathrm{d} \Gamma+\ldots \\
& \ldots . .-\int_{\Omega} \frac{\partial}{\partial \mathrm{x}_{\gamma}}\left(\mathrm{U}_{3}^{3}\right) \mathrm{qd} \Omega
\end{aligned}
$$


The differentiation is performed over the following fundamental solution kernels in equation (8):

$$
\begin{gathered}
\mathrm{T}_{3}^{3}=-\frac{1}{2 \pi} \frac{1}{\mathrm{r}} \frac{\partial \mathrm{r}}{\partial \mathrm{n}} ; \quad \mathrm{U}_{3}^{3}=\frac{1}{8 \pi \mathrm{D}} \mathrm{r}^{2}(\ln (\mathrm{z})-1)-\frac{1}{\pi \mathrm{D} \lambda^{2}(1-v)} \ln (\mathrm{z}) \\
\mathrm{T}_{\beta}^{3}=-\frac{1}{4 \pi}\left[(1+v) \mathrm{n}_{\beta} \ln (\mathrm{z})+(1-v)\left(\frac{\partial \mathrm{r}}{\partial \mathrm{n}} \mathrm{r}_{\beta}-\frac{1}{2} \mathrm{n}_{\beta}\right)\right] ; \quad \mathrm{U}_{\beta}^{3}=-\frac{1}{4 \pi \mathrm{D}} \mathrm{r}\left(\ln (\mathrm{z})-\frac{1}{2}\right) \mathrm{r}_{\beta}
\end{gathered}
$$

The strong singularity is obtained from the differentiation over $\mathrm{T}_{3}{ }^{3}$ whereas $1 / \mathrm{r}$ singularities or logarithmic singularities result from differentiation over other terms. The TDO should be applied over the kernel related to $\mathrm{T}_{3}{ }^{3}$.

$$
\oint_{\Gamma} \frac{\partial}{\partial \mathrm{x}_{\gamma}}\left(\mathrm{T}_{3}^{3}\right) \mathrm{u}_{3} \mathrm{~d} \Gamma=\oint_{\Gamma} \frac{\partial}{\partial \mathrm{x}_{\gamma}}\left(\mathrm{Q}_{\beta}^{3} \mathrm{n}_{\beta}\right) \mathrm{u}_{3} \mathrm{~d} \Gamma=\oint_{\Gamma}\left[\mathrm{D}_{\beta \gamma}\left(\mathrm{Q}_{\beta}^{3}\right)+\mathrm{n}_{\gamma} \frac{\partial}{\partial \mathrm{x}_{\beta}}\left(\mathrm{Q}_{\beta}^{3}\right)\right] \mathrm{u}_{3} \mathrm{~d} \Gamma
$$

$\mathrm{D}_{\mathrm{bm}}(\mathrm{)})$ is the tangential differential operator, which has the following definition:

$$
D_{b m}[f(y)]=n_{b}(y) f_{, m}(y)-n_{m}(y) f_{, b}(y)
$$

The fundamental solution for a unit point force $(\mathrm{i}=3)$ is related to the equilibrium equation (2) in absence of the transverse distributed load q. $Q_{\beta, \beta}{ }^{3}$ is equal to zero outside the source point which is the second term between brackets of equation (9). The final result from equation (9) is next written after the integration by parts to reduce the order of the singularity in the kernel, which changes the application of the TDO to $\mathrm{u}_{3}$, and allowing a discontinuity in the boundary line [6]:

$$
\oint_{\Gamma} \frac{\partial}{\partial \mathrm{x}_{\gamma}}\left(\mathrm{T}_{3}^{3}\right) \mathrm{u}_{3} \mathrm{~d} \Gamma=\oint_{\Gamma} \mathrm{Q}_{\beta}^{3} \mathrm{D}_{\gamma \beta}\left(\mathrm{u}_{3}\right) \mathrm{d} \Gamma+\left[\mathrm{e}_{3 \beta \gamma} \mathrm{Q}_{\beta}^{3} \mathrm{u}_{3}\right]_{0}^{\Gamma}
$$

$\mathrm{e}_{\mathrm{i} \beta \gamma}$ is the permutation symbol.

The term between brackets in equation (10) is cut off when the boundary line is continuous. The final expression for the deflection gradient is given by:

$$
\begin{aligned}
\mathrm{u}_{3, \gamma}= & \oint_{\Gamma}\left(\frac{\partial}{\partial \mathrm{x}_{\gamma}}\left(\mathrm{T}_{\beta}^{3}\right) \mathrm{u}_{\beta}+\mathrm{Q}_{\beta}^{3} \mathrm{D}_{\gamma \beta}\left(\mathrm{u}_{3}\right)-\frac{\partial}{\partial \mathrm{x}_{\gamma}}\left(\mathrm{U}_{\beta}^{3}\right) \mathrm{t}_{\beta}-\frac{\partial}{\partial \mathrm{x}_{\gamma}}\left(\mathrm{U}_{3}^{3}\right) \mathrm{t}_{3}\right) \mathrm{d} \Gamma+\ldots \\
& \ldots . .-\iint_{\Omega} \frac{\partial}{\partial \mathrm{x}_{\gamma}}\left(\mathrm{U}_{3}^{3}\right) \mathrm{qd} \Omega+\left[\mathrm{e}_{3 \beta \gamma} \mathrm{Q}_{\beta}^{3} \mathrm{u}_{3}\right]_{0}^{\Gamma}
\end{aligned}
$$

The algebraic manipulation to introduce the TDO in the BIE for the rotation gradient at an internal point is similar but not equal to that presented for the deflection gradient because the BIE for plates relates the distributed shear and couples with the deflection and rotations in same equation. The forces and displacements are not the same type as in BIEs for two or three-dimensional elasticity and additional care is necessary. The BIE for the rotation gradient is next written using the differentiation in terms of field variables:

$$
\begin{aligned}
\mathrm{u}_{\rho, \gamma}= & \oint_{\Gamma}\left(\frac{\partial}{\partial \mathrm{x}_{\gamma}}\left(\mathrm{T}_{\beta}^{\rho}\right) \mathrm{u}_{\beta}+\frac{\partial}{\partial \mathrm{x}_{\gamma}}\left(\mathrm{T}_{3}^{\rho}\right) \mathrm{u}_{3}-\frac{\partial}{\partial \mathrm{x}_{\gamma}}\left(\mathrm{U}_{\beta}^{\rho}\right) \mathrm{t}_{\beta}-\frac{\partial}{\partial \mathrm{x}_{\gamma}}\left(\mathrm{U}_{3}^{\rho}\right) \mathrm{t}_{3}\right) \mathrm{d} \Gamma+\ldots \\
& \ldots . .-\iint_{\Omega} \frac{\partial}{\partial \mathrm{x}_{\gamma}}\left(\mathrm{U}_{3}^{\rho}\right) \mathrm{q} \mathrm{d} \Omega
\end{aligned}
$$


The fundamental solution kernels of equation (12), where the differentiation is performed, are next written as was done for equation (8):

$$
\begin{aligned}
& \mathrm{T}_{\beta}^{\rho}=-\frac{1}{\pi}\left[\left(\frac{\lambda \mathrm{K}_{1}(\mathrm{z})}{2}+\frac{(1-v)}{4 \mathrm{r}}+\frac{\mathrm{A}(\mathrm{z})}{\mathrm{r}}\right)\left[\left(\delta_{\rho \beta}-2 \mathrm{r}_{\beta} \mathrm{r}_{\rho}\right) \frac{\partial \mathrm{r}}{\partial \mathrm{n}}+\mathrm{n}_{\rho} \mathrm{r}_{\beta}\right]+\ldots\right. \\
&\left.\ldots . .+\frac{(1-v)}{4 \mathrm{r}} \mathrm{n}_{\beta} \mathrm{r}_{\rho}-\frac{\mathrm{A}(\mathrm{z})}{\mathrm{r}}\left(2 \mathrm{r}_{\beta} \mathrm{r}_{\rho} \frac{\partial \mathrm{r}}{\partial \mathrm{n}}-\mathrm{n}_{\beta} \mathrm{r}\right)\right] ; \\
& \mathrm{U}_{\beta}^{\rho}= \frac{1}{(1-v)} \frac{1}{\pi \mathrm{D}}\left(\mathrm{B}(\mathrm{z}) \delta_{\beta \rho}-\mathrm{A}(\mathrm{z}) \mathrm{r}_{\beta} \mathrm{r}_{\rho}\right)-\frac{1}{4 \pi \mathrm{D}}\left[\left(\ln (\mathrm{z})-\frac{1}{2}\right) \delta_{\beta \rho}+\mathrm{r}_{\beta} \mathrm{r}_{\rho}\right] ; \\
& \mathrm{T}_{3}^{\rho}=\frac{1}{2 \pi} \lambda^{2}\left(\mathrm{~B}(\mathrm{z}) \mathrm{n}_{\rho}-\mathrm{A}(\mathrm{z}) \frac{\partial \mathrm{r}}{\partial \mathrm{n}} \mathrm{r}_{\rho}\right) ; \quad \mathrm{U}_{3}^{\rho}=\frac{1}{4 \pi \mathrm{D}} \mathrm{r}\left(\ln (\mathrm{z})-\frac{1}{2}\right) \mathrm{r}_{\rho} .
\end{aligned}
$$

with

$$
\mathrm{z}=\lambda \mathrm{r} ; \quad \mathrm{B}(\mathrm{z})=\mathrm{K}_{0}(\mathrm{z})+\frac{1}{\mathrm{z}}\left[\mathrm{K}_{1}(\mathrm{z})-\frac{1}{\mathrm{z}}\right] ; \quad \mathrm{A}(\mathrm{z})=2 \mathrm{~B}(\mathrm{z})-\mathrm{K}_{0}(\mathrm{z}) .
$$

An expansion for small arguments should be considered for terms containing modified Bessel functions with real arguments $\left(\mathrm{K}_{0}, \mathrm{~K}_{1}\right)$ in the analysis of the singularity. The strong singularity is obtained from the differentiation on $\mathrm{T}_{\beta}{ }^{\rho}$ whereas $1 / \mathrm{r}$ singularities or logarithmic singularities types result from differentiation over other terms. The TDO is applied on the kernel related to $\mathrm{T}_{\beta}{ }^{p}$ of equation (12):

$$
\oint_{\Gamma} \frac{\partial}{\partial x_{\gamma}}\left(T_{\beta}^{\rho}\right) u_{\beta} d \Gamma=\oint_{\Gamma} \frac{\partial\left(M_{\alpha \beta}^{\rho} n_{\alpha}\right)}{\partial x_{\gamma}} u_{\beta} d \Gamma=\oint_{\Gamma}\left[D_{\alpha \gamma}\left(M_{\alpha \beta}^{\rho}\right)+n_{\gamma} \frac{\partial\left(M_{\alpha \beta}^{\rho}\right)}{\partial x_{\alpha}}\right] u_{\beta} d \Gamma
$$

The fundamental solution for a unit couple $(\rho=1,2)$ is related to the equilibrium equation (1). $\mathrm{M}_{\alpha \beta, \alpha}{ }^{\rho}$ is equal to $\mathrm{Q}_{\beta}{ }^{\rho}$ outside the source point, which is a regular function for the unit couple solution [15]. The final result from equation (13) is next written after the integration by parts to reduce the order of the singularity in the kernel, which changes the application of the TDO to $\mathrm{u}_{\beta}$, and allowing a discontinuity over the boundary line [6]:

$$
\oint_{\Gamma} \frac{\partial}{\partial x_{\gamma}}\left(T_{\beta}^{\rho}\right) u_{\beta} d \Gamma=\oint_{\Gamma}\left[M_{\alpha \beta}^{\rho} D_{\gamma \alpha}\left(u_{\beta}\right)+n_{\gamma} Q_{\beta}^{\rho} u_{\beta}\right] d \Gamma+\left[e_{3 \alpha \gamma} M_{\alpha \beta}^{\rho} u_{\beta}\right]_{0}^{\Gamma}
$$

The term between brackets in equation (14) is cut off when the boundary line is continuous. The final expression for the rotation gradient is given by:

$$
\begin{gathered}
\mathrm{u}_{\rho, \gamma}=\oint_{\Gamma}\left(\mathrm{M}_{\alpha \beta}^{\rho} \mathrm{D}_{\gamma \alpha}\left(\mathrm{u}_{\beta}\right)+\mathrm{n}_{\gamma} \mathrm{Q}_{\beta}^{\rho} \mathrm{u}_{\beta}+\frac{\partial}{\partial \mathrm{x}_{\gamma}}\left(\mathrm{T}_{3}^{\rho}\right) \mathrm{u}_{3}-\frac{\partial}{\partial \mathrm{x}_{\gamma}}\left(\mathrm{U}_{\beta}^{\rho}\right) \mathrm{t}_{\beta}+\ldots\right. \\
\left.\ldots . .-\frac{\partial}{\partial \mathrm{x}_{\gamma}}\left(\mathrm{U}_{3}^{\rho}\right) \mathrm{t}_{3}\right) \mathrm{d} \Gamma-\iint_{\Omega} \frac{\partial}{\partial \mathrm{x}_{\gamma}}\left(\mathrm{U}_{3}^{\rho}\right) \mathrm{qd} \Omega+\left[\mathrm{e}_{3 \alpha \gamma} \mathrm{M}_{\alpha \beta}^{\rho} \mathrm{u}_{\beta}\right]_{0}^{\Gamma}
\end{gathered}
$$

The BIE for the distributed shear at internal points can be obtained using the constitutive equation (4) together with equations (7) and (11). 


$$
\begin{aligned}
\mathrm{Q}_{\gamma}= & \mathrm{D} \frac{(1-v)}{2} \lambda^{2}\left\{\oint_{\Gamma}\left[\left(\mathrm{T}_{\beta, \gamma}^{3}-\mathrm{T}_{\beta}^{\gamma}\right) \mathrm{u}_{\beta}+\mathrm{Q}_{\beta}^{3} \mathrm{D}_{\gamma \beta}\left(\mathrm{u}_{3}\right)-\mathrm{T}_{3}^{\gamma} \mathrm{u}_{3}\right] \mathrm{d} \Gamma+\left[\mathrm{e}_{3 \beta \gamma} \mathrm{Q}_{\beta}^{3} \mathrm{u}_{3}\right]_{0}^{\Gamma}\right\}+\ldots \\
& \ldots . .+\oint_{\Gamma}\left(\mathrm{Q}_{\gamma}^{\beta} \mathrm{t}_{\beta}-\mathrm{Q}_{\gamma}^{3} \mathrm{t}_{3}\right) \mathrm{d} \Gamma-\iint_{\Omega} \mathrm{qQ} \mathrm{Q}_{\gamma}^{3} \mathrm{~d} \Omega
\end{aligned}
$$

The algebraic manipulation to get $\mathrm{Q}_{\gamma}{ }^{\beta}$ and $\mathrm{Q}_{\gamma}{ }^{3}$ in equation (16) considered the symmetrical property of $\mathrm{U}_{\beta}{ }^{\alpha}$ and that $\mathrm{U}_{\beta}{ }^{3}$ is $-\mathrm{U}_{3}{ }^{\beta}$. Furthermore, the field decomposition presented in $[15,16]$ can be used to check the symmetry relations notwithstanding the algebraic expressions used for fundamental solutions.

The BIE for bending and twisting moments at internal points is obtained with the constitutive equation (3) together with equation (15). The BIE is next written using the symmetrical property of $\mathrm{U}_{\beta}{ }^{\alpha}$ and that $\mathrm{U}_{\beta}{ }^{3}$ is $-\mathrm{U}_{3}{ }^{\beta}$ :

$$
\begin{aligned}
\mathrm{M}_{\alpha \beta}= & \mathrm{C}_{\alpha \beta \rho \gamma}\left\{\oint_{\Gamma}\left(\mathrm{M}_{\alpha \beta}^{\rho} \mathrm{D}_{\gamma \alpha}\left(\mathrm{u}_{\beta}\right)+\mathrm{n}_{\gamma} \mathrm{Q}_{\beta}^{\rho} \mathrm{u}_{\beta}+\mathrm{T}_{3, \gamma}^{\rho} \mathrm{u}_{3}\right) \mathrm{d} \Gamma+\left[\mathrm{e}_{3 \alpha \gamma} \mathrm{M}_{\alpha \beta}^{\rho} \mathrm{u}_{\beta}\right]_{0}^{\Gamma}\right\}+\ldots \\
& \ldots . .+\oint_{\Gamma}\left(\mathrm{M}_{\alpha \beta}^{3} \mathrm{t}_{3}-\mathrm{M}_{\alpha \beta}^{\gamma} \mathrm{t}_{\gamma}\right) \mathrm{d} \Gamma+\iint_{\Omega} \mathrm{q} \mathrm{M}_{\alpha \beta}^{3} \mathrm{~d} \Omega
\end{aligned}
$$

with

$$
\mathrm{C}_{\alpha \beta \rho \gamma}=\mathrm{D} \frac{(1-v)}{2}\left(\delta_{\alpha \rho} \delta_{\beta \gamma}+\delta_{\alpha \gamma} \delta_{\beta \rho}+\frac{2 v}{1-v} \delta_{\alpha \beta} \delta_{\rho \gamma}\right)
$$

The integrals of equations (16) and (17) are regular for internal points and exhibit singularities or singularity type of order $1 / \mathrm{r}$ when the field point approaches the collocation point. The BIEs for distributed shear and moments at a boundary point is defined as the limiting form of the corresponding BIE at an internal point when it is led to a point on the boundary. Equations (16) and (17) are next written for the collocation point on a smooth boundary:

$$
\begin{aligned}
& \frac{1}{2} \mathrm{Q}_{\gamma}= \mathrm{D} \frac{(1-v)}{2} \lambda^{2}\left\{\oint_{\Gamma}\left[\left(\mathrm{T}_{\beta, \gamma}^{3}-\mathrm{T}_{\beta}^{\gamma}\right) \mathrm{u}_{\beta}+\mathrm{Q}_{\beta}^{3} \mathrm{D}_{\gamma \beta}\left(\mathrm{u}_{3}\right)-\mathrm{T}_{3}^{\gamma} \mathrm{u}_{3}\right] \mathrm{d} \Gamma+\ldots\right. \\
&\left.\ldots . .+\left[\mathrm{e}_{3 \beta \gamma} \mathrm{Q}_{\beta}^{3} \mathrm{u}_{3}\right]_{0}^{\Gamma}\right\}+\oint_{\Gamma}\left(\mathrm{Q}_{\gamma}^{\beta} \mathrm{t}_{\beta}-\mathrm{Q}_{\gamma}^{3} \mathrm{t}_{3}\right) \mathrm{d} \Gamma-\iint_{\Omega} \mathrm{q} \mathrm{Q}_{\gamma}^{3} \mathrm{~d} \Omega \\
& \frac{1}{2} \mathrm{M}_{\alpha \beta}=\mathrm{C}_{\alpha \beta \rho \gamma}\left\{\oint_{\Gamma}\left(\mathrm{M}_{\alpha \beta}^{\rho} \mathrm{D}_{\gamma \alpha}\left(\mathrm{u}_{\beta}\right)+\mathrm{n}_{\gamma} \mathrm{Q}_{\beta}^{\rho} \mathrm{u}_{\beta}+\mathrm{T}_{3, \gamma}^{\rho} \mathrm{u}_{3}\right) \mathrm{d} \Gamma+\left[\mathrm{e}_{3 \alpha \gamma} \mathrm{M}_{\alpha \beta}^{\rho} \mathrm{u}_{\beta}\right]_{0}^{\Gamma}\right\}+\ldots \\
& \ldots . .+\oint_{\Gamma}\left(\mathrm{M}_{\alpha \beta}^{3} \mathrm{t}_{3}-\mathrm{M}_{\alpha \beta}^{\gamma} \mathrm{t}_{\gamma}\right) \mathrm{d} \Gamma+\iint_{\Omega} \mathrm{qM}_{\alpha \beta}^{3} \mathrm{~d} \Omega
\end{aligned}
$$

It is important to note the continuity requirement for the derivative of the displacement function at the collocation point. The BIEs for tractions are obtained from equation (18) and (19) when the distributed shear tensor and the moment tensor at the collocation point on the boundary point $\mathrm{x}$ ' are multiplied by direction cosines of the outward normal at this point (n'), i.e. the Cauchy formula: 


$$
\begin{aligned}
\frac{1}{2} \mathrm{t}_{3}\left(\mathrm{x}^{\prime}\right)=\mathrm{D} \frac{(1-v)}{2} \lambda^{2} \mathrm{n}_{\gamma}^{\prime}\left(\mathrm{x}^{\prime}\right)\left\{\oint_{\Gamma}\left[\left(\mathrm{T}_{\beta, \gamma}^{3}-\mathrm{T}_{\beta}^{\gamma}\right) \mathrm{u}_{\beta}+\mathrm{Q}_{\beta}^{3} \mathrm{D}_{\gamma \beta}\left(\mathrm{u}_{3}\right)-\mathrm{T}_{3}^{\gamma} \mathrm{u}_{3}\right] \mathrm{d} \Gamma+\ldots\right. \\
\left.\ldots . .+\left[\mathrm{e}_{3 \beta \gamma} \mathrm{Q}_{\beta}^{3} \mathrm{u}_{3}\right]_{0}\right\}+\mathrm{n}_{\gamma}^{\prime}\left(\mathrm{x}^{\prime}\right) \underset{\Gamma}{\oint}\left(\mathrm{Q}_{\gamma}^{\beta} \mathrm{t}_{\beta}-\mathrm{Q}_{\gamma}^{3} \mathrm{t}_{3}\right) \mathrm{d} \Gamma-\mathrm{n}_{\gamma}^{\prime}\left(\mathrm{x}^{\prime}\right) \iint_{\Omega} \mathrm{q} \mathrm{Q}_{\gamma}^{3} \mathrm{~d} \Omega \\
\frac{1}{2} \mathrm{t}_{\beta}\left(\mathrm{x}^{\prime}\right)=\mathrm{C}_{\alpha \beta \beta \gamma} \mathrm{n}_{\alpha}^{\prime}\left(\mathrm{x}^{\prime}\right)\left\{\oint_{\Gamma}\left(\mathrm{M}_{\alpha \beta}^{\rho} \mathrm{D}_{\gamma \alpha}\left(\mathrm{u}_{\beta}\right)+\mathrm{n}_{\gamma} \mathrm{Q}_{\beta}^{\rho} \mathrm{u}_{\beta}+\mathrm{T}_{3, \gamma}^{\rho} \mathrm{u}_{3}\right) \mathrm{d} \Gamma+\ldots\right. \\
\left.\ldots . .+\left[\mathrm{e}_{3 \alpha \gamma} \mathrm{M}_{\alpha \beta}^{\rho} \mathrm{u}_{\beta}\right]_{0}^{\Gamma}\right\}+\mathrm{n}_{\alpha}^{\prime}\left(\mathrm{x}^{\prime}\right)\left[\oint_{\Gamma}\left(\mathrm{M}_{\alpha \beta}^{3} \mathrm{t}_{3}-\mathrm{M}_{\alpha \beta}^{\gamma} \mathrm{t}_{\gamma}\right) \mathrm{d} \Gamma+\iint_{\Omega} \mathrm{qM}_{\alpha \beta}^{3} \mathrm{~d} \Omega\right]
\end{aligned}
$$

The collocation points must be positioned to satisfy the continuity requirements of BIEs for tractions. The continuity of the displacement function at $x^{\prime}$ is the necessary condition for the generalized displacement BIE and it is satisfied when the collocation point is placed at the ends of the boundary element or inside the element. The continuities of the displacement and rotations derivatives at $\mathrm{x}^{\prime}$ are required for traction BIEs and they can be satisfied when the collocation point is placed inside the boundary element.

\section{Numerical implementation}

Linear mapping functions were used to represent displacements and efforts in the boundary elements. The same mapping function was used for conformal and non-conformal interpolations with nodal parameters positioned at the ends of the elements. The collocation points were shifted to the interior of the element at a distance of a sixth part of its length starting from the end. The collocation points were always positioned in the boundary line with the position ( $\xi$ '), in the range $(-1,1)$ : i) $\xi^{\prime}=-0.67$ for continuous elements; ii) $\xi^{\prime}=-0.67$ and $\xi^{\prime}=+0.67$ for discontinuous elements. Analytical expressions were used to evaluate singular integrals with the Cauchy principal value sense whereas the Gauss-Legendre scheme was used for regular integrals. An expansion for small arguments was considered for terms containing modified Bessel functions with real arguments $\left(\mathrm{K}_{0}, \mathrm{~K}_{1}\right)$ [17]. The diagonal terms were directly obtained using the mapping function and the collocation point position on the element. The numerical implementation for the tangential differential operator considered the differentiation of the mapping function according to the following relation [18]:

$$
D_{b m}\{f[y(\xi)]\}=n_{b}(y) f_{, m}(y)-n_{m}(y) f_{, b}(y)=e_{3 \gamma p} \frac{1}{|J|} \frac{d f}{d \xi}
$$

$\mathrm{J}$ is the Jacobian of the transformation and $\xi$ is the intrinsic coordinate to perform the integration on the element.

\section{Example - the torsion of a cube}

The problem was analyzed with traction BIEs using the TDO, equations (20) and (21), replacing the displacement BIEs (equation (7)) for the solution with 
boundary element method. The torsion of cube is used to show the precision of the formulation and justify the adopted position for the collocation point. A mesh with 128 linear discontinuous elements (256 nodes) along the sides was used. The results were compared with those presented in [1] where the traction BIE containing the strong singularity was used in a mesh containing 128 quadratic discontinuous elements (384 nodes) and with those presented by Weeën [14], that employed symmetry conditions in the solution with the displacement BIE using 8 quadratic continuous elements (16 nodes). The cube has the side length equals to $2 \mathrm{a}$, two opposite faces are free of stress and other faces are under torsion according to Saint-Venant hypotheses (free warping). The generalized displacements were used to introduce torsion:

$$
\begin{gathered}
\mathrm{u}_{3}=\varphi \mathrm{ax}_{2} \\
\mathrm{u}_{2}=\varphi \mathrm{a}
\end{gathered}
$$

$\varphi$ is the prescribed rotation angle

Table 1: Relative values of plate rotations in the normal $\operatorname{direction}\left(\psi_{\mathrm{n}}\left(\mathrm{x}_{2}\right) / \psi_{\mathrm{n}}(\mathrm{a})\right)$.

\begin{tabular}{|c|c|c|c|c|c|c|}
\hline$\left(\mathrm{x}_{2} / \mathrm{a}\right)$ & {$[12]$} & {$[1]$} & {$[14]$} & $\xi=0.5$ & $\xi=0.67$ & $\xi=0.75$ \\
\hline 1.00 & 1.000 & 1.000 & 1.001 & 1.000 & 1.000 & 1.000 \\
\hline 0.75 & -0.055 & -0.051 & -0.053 & -0.096 & -0.058 & -0.034 \\
\hline 0.50 & -0.387 & -0.382 & -0.386 & -0.428 & -0.389 & -0.366 \\
\hline 0.25 & -0.292 & -0.290 & -0.290 & -0.317 & -0.294 & -0.280 \\
\hline 0.00 & 0.000 & 0.000 & 0.000 & 0.000 & 0.000 & 0.000 \\
\hline
\end{tabular}

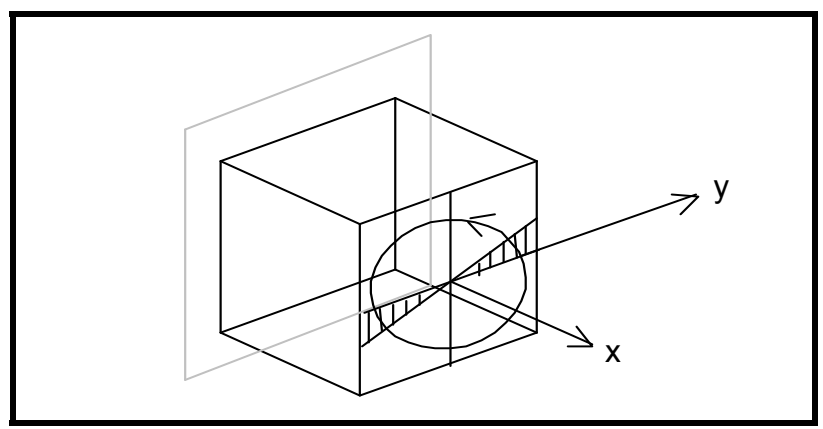

Figure 1: $\quad$ Torsion of a cube.

The greatest differences in results to values obtained by Reissner [12] appeared for plate rotations in the normal direction because these values are indirectly related with prescribed displacements. These rotations were used to qualify the collocation point position. The values for distributed shear and twisting moments are directly related to the constrained displacements, the differences to values obtained by Reissner [12] were low and they cannot be used to qualify the collocation point position or the boundary element solution. 
Table 2: $\quad$ Relative values of distributed shear $\left(\mathrm{Q}_{\mathrm{n}}\left(\mathrm{x}_{2}\right) / \mathrm{Q}_{\mathrm{n}}(\mathrm{a})\right)$.

\begin{tabular}{|c|c|c|c|c|c|c|}
\hline$\left(\mathrm{x}_{2} / \mathrm{a}\right)$ & {$[12]$} & {$[1]$} & {$[14]$} & $\xi=0.5$ & $\xi=0.67$ & $\xi=0.75$ \\
\hline 1.00 & 1.000 & 1.000 & 0.999 & 1.000 & 1.000 & 1.000 \\
\hline 0.75 & 0.638 & 0.638 & 0.638 & 0.640 & 0.640 & 0.636 \\
\hline 0.50 & 0.377 & 0.377 & 0.377 & 0.377 & 0.378 & 0.375 \\
\hline 0.25 & 0.174 & 0.174 & 0.174 & 0.175 & 0.175 & 0.174 \\
\hline 0.00 & 0.000 & 1.000 & 0.000 & 0.000 & 0.000 & 0.000 \\
\hline
\end{tabular}

Table 3: $\quad$ Relative values of twisting moments $\left(\mathrm{M}_{\mathrm{t}}(0) / \mathrm{M}_{\mathrm{t}}\left(\mathrm{x}_{2}\right)\right)$.

\begin{tabular}{|c|c|c|c|c|c|c|}
\hline$\left(\mathrm{x}_{2} / \mathrm{a}\right)$ & {$[12]$} & {$[1]$} & {$[14]$} & $\xi=0.5$ & $\xi=0.67$ & $\xi=0.75$ \\
\hline 1.00 & 0.000 & 0.000 & 0.035 & 0.000 & 0.000 & 0.000 \\
\hline 0.75 & 0.485 & 0.485 & 0.469 & 0.487 & 0.486 & 0.484 \\
\hline 0.50 & 0.785 & 0.785 & 0.809 & 0.786 & 0.786 & 0.785 \\
\hline 0.25 & 0.948 & 0.948 & 0.938 & 0.949 & 0.948 & 0.948 \\
\hline 0.00 & 1.000 & 1.000 & 1.018 & 1.000 & 1.000 & 1.000 \\
\hline
\end{tabular}

\section{Conclusions}

The use of the tangential differential operator in traction BIE for plate bending models considering the shear deformation effect allowed the order reduction of the singularities without reducing the accuracy of the boundary element method. The use of fundamental solutions having singularity at the source point is the main request to apply the TDO with the integration by parts. The results have agreed with those obtained in [12, 1 and 14]. Thus, the same efficiency shown for two dimensional problems of the linear fracture mechanics in [6] and for three dimensional elasticity problems in [7] was also shown for plate bending models including the shear deformation effect. Furthermore, the use of derivatives of the adopted shape function for displacement without using other interpolation for TDO was an interesting alternative without reducing the expected precision. The next step will be the study of fracture problems in plate bending using the DBEM with the TDO.

\section{Acknowledgement}

The author is grateful to CNPq for support for the development of research on plates.

\section{References}

[1] Rashed YF, Aliabadi MH, Brebbia CA. Hypersingular boundary element formulation for Reissner plates. Int J Solids Struct, 35(18), 2229-49, 1998. 
[2] Guiggiani, M. Hypersingular formulation for boundary stresses evaluation. Eng. Anal. Bound. Elem., 14, 169-179, 1994.

[3] Kupradze, V.D., Three-dimensional problems of the mathematical theory of elasticity and thermoelasticity. North Holland, 1979.

[4] Sladek, J.; Sladek, V., Three-dimensional curved crack in an elastic body, Int. J. Solids Struct., 19, 425-436, 1983.

[5] Bonnet, M. Boundary Integral Equation Methods for Solids and Fluids, John Wiley \& Sons Ltd, 1999.

[6] Palermo, Jr., L., Almeida, L.P.C.P.F. and Gonçalves, P.C., The Use of the Tangential Differential Operator in the Dual Boundary Element Equation, Structural Durability \& Health Monitoring, vol.2, no.2, pp.123-130, Tech Science Press, 2006.

[7] Palermo, Jr., L., Almeida, L.P.C.P.F., On the Use of the Tangential Differential Operator in the Traction Boundary Integral Equation of the Dual Boundary Element Method for Three Dimensional Problems, ICCES, vol.7, no.2, pp.83-87, 2008.

[8] Gonçalves, P.C., Uma Alternativa para a Solução de Problemas de Elasticidade pelo Método dos Elementos de Contorno, MS of Eng. Dissertation (in Portuguese), University of Campinas, 2006.

[9] Almeida, L.P.C.P.F.; Palermo Jr., L. On the Implementation of the Two Dimensional Dual Boundary Element Method for Crack Problems, 5th Int. Conference on Boundary Elements Techniques, Lisboa, Portugal, 2004.

[10] Love A.E.H.. A treatise on the mathematical theory of elasticity, 4th ed. New York, Dover Publications; 1944.

[11] Palermo Jr., L., Gonçalves, P.C., Figueiredo, L.G., A simple implementation of the dual boundary element method using the tangential differential operator for plane problems, BEM 32, Editor C.A. Brebbia, 2010.

[12] Reissner, E., The Effect of Transverse Shear Deformation on the Bending of Elastic Plates, Journal of Applied Mechanics, 1945.

[13] Mindlin, R.D., Influence of rotatory inertia and shear on flexural motions of isotropic elastic plates, Journal of Applied Mechanics, 1951.

[14] Weeën, F., Application of the boundary integral equation method to Reissner's plate model, International Journal for Numerical Methods in Engineering, vol. 18, 1-10, 1982 .

[15] Palermo Jr., L., A strategy to perform the Reissner-Mindlin's theory, BEM 24, Editors C.A. Brebbia, A. Tadeu, V. Popov, 2002.

[16] Palermo Jr., L., Plate Bending analysis using the classical or the ReissnerMindlin models, Engineering Analysis with Boundary Elements, 2003.

[17] Abramowitz M, Stegun I. Handbook of mathematical functions, New York: Dover Publications; 1972.

[18] Kane, J. H., Boundary Element Analysis in engineering mechanics. Prentice Hall, 1994. 